\title{
Factors Affecting Occupational Burnout Among Nurses Including Job Satisfaction, Life Satisfaction, and Life Orientation: A Cross-Sectional Study
}

\author{
Agnieszka Zborowska (D) \\ Piotr Jerzy Gurowiec $\mathbb{D}^{2}$ \\ Agnieszka Młynarska iD ${ }^{3,4}$ \\ Izabella Uchmanowicz (D) 1,5 \\ 'Department of Clinical Nursing, \\ Wroclaw Medical University, Wroclaw, \\ Poland; ${ }^{2}$ Institute of Health Sciences, \\ University of Opole, Opole, Poland; \\ ${ }^{3}$ Department of Gerontology and \\ Geriatric Nursing, Medical University of \\ Silesia, Katowice, Poland; ${ }^{4}$ Department of \\ Electrocardiology, Upper Silesian Heart \\ Centre, Katowice, Poland; ${ }^{5}$ Centre for \\ Heart Diseases, Wroclaw Medical \\ University, Wroclaw, Poland
}

Purpose: Everyday nursing practice under demanding conditions, high mental and physical strain may result in occupational burnout. There is still a need for studies on the comprehensive identification of burnout among nursing staff. This study aimed to evaluate factors affecting occupational burnout among nurses, including job satisfaction, life satisfaction, and dispositional optimism.

Methods: The study involved 625 survey participants with a mean age of 49.47 years and was conducted between January and December 2018. The inclusion criteria were job experience $>1$ year, current nurse work activity, and written informed consent to participate in the study. The study used standardized research tools such as Maslach Burnout Inventory (MBI), Life Orientation Test-Revised version (LOT-R), Satisfaction with Life Scale (SWLS), and Satisfaction with Job Scale (SWJS). Also, sociodemographic data were collected using a self-developed questionnaire. The STROBE guidelines were followed.

Results: Emotional exhaustion (EE) was significantly greater in respondents with medium life satisfaction (SWLS). Also, EE was significantly greater in pessimists and those with a neutral orientation than in optimists (LOT-R). Housing conditions and family-related problems were significant factors differentiating depersonalization (DEP). Reduced personal accomplishment (PA) was more significant in respondents with a bachelor's degree than in those with a master's degree. Also, decreased PA was significantly greater in respondents with low life satisfaction than those with medium life satisfaction. Life satisfaction and life orientation were significant factors differentiating job dissatisfaction among the healthrelated and psychological variables.

Conclusion: Burnout was found to be related to individual, interpersonal and organizational feelings. There was an interrelationship between personality traits, where a higher level of occupational burnout was found among nurses with a pessimist attitude. Health programs should be implemented to identify and eliminate burnout through mental health support, improved communication skills, optimized teamwork, and evidence-based interventions.

Keywords: occupational burnout, emotional exhaustion, job satisfaction, life satisfaction, dispositional optimism, nurses

\section{Introduction}

Close contact with the sick and suffer people makes nursing one of the professions of great social importance, particularly at risk of workload and subsequent occupational burnout. The definition of occupational burnout proposed by Pines and Aronson $^{1}$ defines the phenomenon as a state of physical, emotional, and mental exhaustion caused by prolonged involvement in emotionally taxing situations. It is
Correspondence: Izabella Uchmanowicz Department of Clinical Nursing, Faculty of Health Sciences, Wroclaw Medical University, Bartla 5, Wroclaw, 5I-618, Poland

Tel +48 7I 7841805

$\mathrm{Fax}+48713459324$

Email izabella.uchmanowicz@umed.wroc.pl 
essential to distinguish between occupational burnout and stress, as stress may result in depression, routine, fatigue but not burnout. ${ }^{2}$ Accordingly, burnout is a response to unmet job expectations.

The current version of the job demands-resources (JD-R) model developed by Demerouti et $\mathrm{al}^{3}$ assumes that high job demands (negative physical or emotional stressors) lead to strain and impaired health. In contrast, high resources (positive physical, social, or organizational factors) lead to increased motivation and higher productivity at work. In the case of nurses working under demanding conditions, high mental and physical strain and the need to change the circadian rhythm may result in occupational burnout syndrome. ${ }^{4}$

Such negative factors as time pressure, heavy workload, stressful work conditions, multiple work responsibilities, emotional strain, and difficult relationships with other team members can also be listed as job demands affecting life satisfaction and developing burnout in nursing

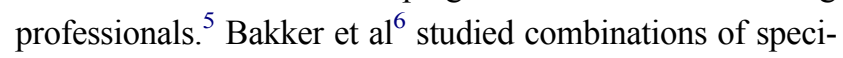
fic job demands and resources and confirmed that exhaustion and cynicism produce the highest levels of burnout, as well as reduced professional efficacy hypothesis was rejected.

Professional competencies are thought to play the most important role in occupational burnout; thus, it is a consequence of competence deficiencies at work. ${ }^{7}$ According to one hypothesis, occupational burnout occurs not only as a result of stress but also as the stress undefined by one's remedy activities. ${ }^{8}$ It was found that nurses were very wary of new competencies and did not feel well prepared for new tasks. The level of life satisfaction and occupational burnout among the surveyed nurses significantly affects their readiness to perform their duties. ${ }^{9}$

The most frequently cited theory concerning occupational burnout is the concept described by Maslach and Jackson $^{10}$ in the literature. According to this theory, occupational burnout is a psychological syndrome of emotional exhaustion, depersonalization, and decreased personal accomplishment, which occurs in persons who work with people in a specific way. ${ }^{11}$ Years later, Maslach ${ }^{12}$ developed a more general concept of occupational burnout, describing burnout as the result of an abnormal relationship between an individual and their environment. According to Maslach and Leiter, ${ }^{13}$ mismatching between an employee and a work unit is also a problem.

Woo et $\mathrm{al}^{14}$ conducted the most current systematic review and meta-analysis to investigate the prevalence of burnout symptoms among nurses, including 45,539 nurses (mean age 25.8-47.0 years) of multiple specialties from 49 countries worldwide. It was found that the global prevalence of burnout symptoms among nurses was $11.23 \%$, and the highest prevalence rate of $14.36 \%$ was found in intensive care units. There is a difference in occupational burnout among nurses regarding the group of patients who are cared for by them in daily clinical practice. It was proved that the most affected dimension of occupational burnout in gynecology wards was a low personal achievement of $44 \%,{ }^{15}$ while emotional exhaustion of $37 \%$ prevailed in pediatric oncology wards. ${ }^{16}$ Depersonalisation was the least affected by burnout in medical wards $-24 \%{ }^{17}$

In terms of occupational burnout, special attention was paid to emotional exhaustion, which is treated as one of the first symptoms of the phenomenon in question. In their systematic review, Salvagioni et al ${ }^{18}$ showed that the most common physical consequences of occupational burnout included insomnia, depressive symptoms, use of psychotropic medications and antidepressants, hospitalization due to mental disorders, psychological symptoms of malaise. In contrast, job dissatisfaction, absenteeism, disability benefit, job demands, job resources, and presenteeism were identified as professional consequences.

The relationship between occupational burnout and helping professions was documented in available academic research. ${ }^{19}$ The literature particularly emphasizes that this phenomenon most frequently affects healthcare professionals, especially nurses. ${ }^{11}$ According to Zhang et al, ${ }^{20}$ comprehensive interventions based on current knowledge are needed to reduce occupational burnout in healthcare professionals, especially doctors and nurses.

Dall'Ora et $\mathrm{al}^{21}$ observed that incomplete measurement of occupational burnout and limited research concerning some dependencies mean that the causes and consequences of occupational burnout cannot be reliably identified and distinguished. Consequently, it is difficult to use the evidence to develop interventions aiming to reduce occupational burnout among nurses. To date, a limited number of studies undertake a comprehensive identification of the scale of the problem of occupational burnout among nurses, taking into account the assessment of job satisfaction, life satisfaction, and dispositional optimism. Hence, this study aimed to evaluate the factors affecting occupational burnout among nurses, considering job satisfaction, life satisfaction, and dispositional optimism. 


\section{Materials and Methods}

\section{Participants and Settings}

The study was conducted among female and male nurses recruited by convenience sampling between January and December 2018 who attended specialist training courses at the European Centre for Postgraduate Education in Wroclaw (Poland). The study involved 625 survey participants aged 24-67; the mean age of the respondents was 49.47 years. The inclusion criteria were job tenure $>$ 1 year, current nurse work activity, and written informed consent to participate in the study. Participation in the study was voluntary and anonymous. The STROBE (The Strengthening the Reporting of Observational Studies in Epidemiology) guidelines were followed.

\section{Research Tools}

The study applied the questionnaire method. A selfdeveloped questionnaire was used to collect sociodemographic, health-related, and occupational data. A standardized research tools were used, such as (1) Maslach Burnout Inventory (MBI), (2) Life Orientation Test-Revised version (LOT-R), (3) Satisfaction with Life Scale (SWLS), and (4) Satisfaction with Job Scale (SWJS).

\section{Self-Developed Survey}

A self-developed questionnaire was used to collect sociodemographic (data such as age, sex, marital status, education, place of residence, housing conditions, family situation), health-related (chronic illnesses, stimulants used, ways to cope with work-related stress and strain), and occupational (net monthly income, job tenure, postgraduate education, number of places of work, main place of work, time spent at work, amount of sleep, amount of free time, nature of work, system of work, number of patients) data.

\section{Maslach Burnout Inventory (MBI)}

MBI was developed by Maslach and Jackson ${ }^{10}$ in 1981. The presented study used the Polish adaptation of the tool by Pasikowski. ${ }^{22}$ The tool consists of 22 items related to three domains: emotional exhaustion (EE), depersonalization (DEP), and decreased personal accomplishment (PA). Scores on each of these domains are expressed on a 0-100 scale, where a higher score indicates a higher level of occupational burnout. Moreover, the total MBI score, which is the mean of these three domains, is also calculated. Higher scores on the EE and DEP domains and lower scores on the PA domain conclude that a surveyed person suffers from occupational burnout. The MBI was validated in Polish by Pasikowski ${ }^{22}$ and achieved Cronbach's alpha coefficients for the scales: EE of 0.85, DEP of 0.60, and PA of 0.76 .

\section{Life Orientation Test-Revised Version (LOT-R)} LOT-R was developed by Scheier et $\mathrm{al}^{23}$ in 1994 . However, it was adapted to Polish conditions by Poprawa and Juczyński. ${ }^{24}$ The tool consists of 10 statements, 6 of which have diagnostic value. This tool is used for investigating dispositional optimism. It is designed for both healthy and sick individuals. The internal consistency of the LOT-R test was determined on a sample of 174 individuals aged 20-55 years, for which Cronbach's alpha coefficient was 0.76 . The internal consistency of the Polish version is similar to that of the original version of the LOT-R test. ${ }^{24}$

\section{Satisfaction with Life Scale (SWLS)}

SWLS was developed by Diener et al. ${ }^{25}$ In turn, the Polish translation and adaptation were prepared by Juczyński. ${ }^{26}$ This tool consists of 5 statements rated on a seven-point scale. The respondent assesses the extent to which each statement relates to their past life. The measurement result is a total index of subjective life satisfaction. The scores of the original SWLS showed an internal consistency of 0.87 and demonstrated sufficient sensitivity to be a potentially valuable tool for detecting changes in life satisfaction. ${ }^{25}$ In Polish validation, the reliability of the SWLS was high with a Cronbach alpha value of 0.891 , and all scale questions correlated positively with each other with a Pearson ratio of 0.529-0.797. ${ }^{27}$

\section{Satisfaction with Job Scale (SWJS)}

SWJS is a tool used for measuring total job satisfaction. This tool consists of five statements concerning the sphere of work. Responses to each statement are given on a seven-point Likert scale, where 1 means "I strongly disagree" and 7 "I strongly agree." Scores range between 5-35, with higher scores indicating greater job satisfaction. There are no standards for the SWJS questionnaire to determine whether a respondent's score is high or low. The midpoint of the SWJS scale is 20 points (4 points for each question), which indicates that a respondent is neither satisfied nor dissatisfied with their job. ${ }^{28}$ The internal reliability and consistency of the SWJS tool is high, with a Cronbach's alpha coefficient of $0.864 .{ }^{28}$

\section{Data Collection}

Packets with the questionnaires used in this study were successively distributed to participants of qualifying and 
specialist courses at the European Centre for Postgraduate Education in Wroclaw (Poland). Participation in the study was voluntary, and respondents were assured of complete anonymity. The researcher, Agnieszka Zborowska, was personally responsible for distributing the questionnaires and collecting them after they had been filled out by nurses. Then, the completeness of returned questionnaires was verified. Out of all the questionnaires filled out by the respondents, 95\% of complete questionnaires were collected and finally included in the analysis (only 5\% of questionnaires dropped out to be incomplete).

\section{Ethical Considerations}

The study design was accepted and approved by the Bioethics Committee of the Wroclaw Medical University (KB-208/2018). Study participants were informed about the study aim and conduct, as well as they gave their informed consent to participate in the survey. Standardized survey questionnaires were used for conducting the study. The study was carried out following the Declaration of Helsinki and Good Clinical Practice guidelines.

\section{Sample Size}

To perform a sample size calculation, a formula designed for nursing observational studies was used, namely: $\mathrm{n}=$ $\{(Z 1-\alpha / 2) 2(p)(q)\} / d 2$, where $40 \%$ was taken as the prevalence rate of depression among nurses by Sharma et al. ${ }^{29}$ Based on data from the Polish Supreme Chamber of Nurses and Midwives, there were nearly 261,000 nurses employed in Poland when the study was performed. It was determined that a minimum sample size of 334 completed questionnaires was needed to achieve a $95 \%$ confidence interval and appropriate level of significance of $5 \%$ for $\alpha / P$ $=0.05$. Therefore, the final statistical analysis in our study involved complete data obtained from 625 nurses.

\section{Data Analyses}

Qualitative variables were compared using the chisquared test (with Yates' correction for $2 \times 2$ tables) or Fisher's exact test. As analyzed quantitative variables were not normally distributed, the comparisons were made using the Mann-Whitney $U$-test for two groups and the Kruskal-Wallis test for three or more groups. Subsequently, the post hoc analysis was performed using the Dunn's test. The normality of the variable distribution was verified using the Shapiro-Wilk test. The MBI score as well as the results of LOT-Rm
SWLS, and SWJS questionnaires did not have normal distribution. Therefore, the Spearman correlation coefficient was used for analysis. The correlation strength was interpreted in accordance with the following scheme: $|\mathrm{r}| \geq$ 0.9 - very strong correlation; $0.7 \leq|\mathrm{r}|<0.9$ - strong correlation; $0.5 \leq|\mathrm{r}|<0.7$ - moderately strong correlation; $0.3 \leq|\mathrm{r}|<0.5$ - weak correlation; $|\mathrm{r}|<0.3$ - very weak correlation (negligible). A multivariate linear regression analysis of the independent effect of many variables on the qualitative variable (MBI score) was also performed. Only variables that appeared to have a significant effect in univariate analyses were included in the multivariate analysis. The R2 determination coefficient assessed the resulting quality of the model. The results are presented in the form of regression model parameters with a 95\% confidence interval. The analysis assumed a materiality level of 0.05 . Thus, all p-values below 0.05 were interpreted as indicating significant dependencies. The analysis was conducted using the R program, version $3.4 .3 .^{30}$

\section{Results}

\section{Characteristics of the Study Group}

A total of 604 female nurses (96.64\%) and 21 male nurses $(3.36 \%)$ participated in the survey. The largest group involved nurses aged 41-50 years (48.80\%). The vast majority of respondents were in a relationship (81.12\%). Nearly half of respondents earned a bachelor's degree $(44.48 \%)$, while one in four respondents earned a master's degree (25.12\%). Most respondents lived in a city $>100,000$ inhabitants $(50.24 \%)$, in good housing conditions $(84.16 \%)$, and they declared support from family $(73.92 \%)$. Detailed characteristics of surveyed nurses are shown in Table 1.

The detailed characteristics of surveyed nurses in terms of life satisfaction (SWJS) and job satisfaction (SWLS), life orientation (LOT-R), and level of occupational burnout (MBI) are shown in Table 2.

\section{Correlation Between Burnout and Job Satisfaction}

Analysis of Spearman correlation coefficient showed the positive correlations between the SWJS score and the DEP domain $(\mathrm{r}=0.257 ; \mathrm{p}<0.001)$ as well as a negative correlation between SWJS score and lack of PA $(r=-0.654$; $\mathrm{p}<0.001)$ and total MBI score $(\mathrm{r}=-0.354 ; \mathrm{p}<0.001)$. There was no significant correlation regarding the EE 
Table I Characteristics of the Study Group

\begin{tabular}{|c|c|c|c|}
\hline \multicolumn{2}{|c|}{ Sociodemographic Variables } & \multirow{2}{*}{$\frac{\mathbf{N}}{130}$} & \multirow{2}{*}{$\begin{array}{l}\% \\
20.80 \%\end{array}$} \\
\hline Age (years) & $23-30$ & & \\
\hline & $31-40$ & 88 & $14.08 \%$ \\
\hline & $4 I-50$ & 305 & $48.80 \%$ \\
\hline & $5 I-60$ & 92 & $14.72 \%$ \\
\hline & $>61$ & 4 & $0.64 \%$ \\
\hline & No answer & 6 & $0.96 \%$ \\
\hline \multirow[t]{3}{*}{ Sex } & Women & 604 & $96.64 \%$ \\
\hline & Men & 19 & $3.04 \%$ \\
\hline & No answer & 2 & $0.32 \%$ \\
\hline \multirow[t]{3}{*}{ Marital status } & In a relationship & 507 & $81.12 \%$ \\
\hline & Single & 108 & $17.28 \%$ \\
\hline & No answer & 10 & $1.60 \%$ \\
\hline \multirow[t]{6}{*}{ Education } & Secondary medical school & 136 & $21.76 \%$ \\
\hline & Medical vocational school & 41 & $6.56 \%$ \\
\hline & Bachelor's degree & 278 & $44.48 \%$ \\
\hline & Master's degree & 157 & $25.12 \%$ \\
\hline & Other & 7 & $1.12 \%$ \\
\hline & No answer & 6 & $0.96 \%$ \\
\hline \multirow[t]{4}{*}{ Place of residence } & City $>100,000$ inhabitants & 314 & $50.24 \%$ \\
\hline & City $<100,000$ inhabitants & 165 & $26.40 \%$ \\
\hline & Rural area & 139 & $22.24 \%$ \\
\hline & No answer & 7 & $1.12 \%$ \\
\hline \multirow[t]{4}{*}{ Housing conditions } & Good & 526 & $84.16 \%$ \\
\hline & Poor & 7 & $1.12 \%$ \\
\hline & Sufficient & 85 & $13.60 \%$ \\
\hline & No answer & 7 & $1.12 \%$ \\
\hline \multirow[t]{7}{*}{ Family situation } & My family supports me; I have no family-related problems & 462 & $73.92 \%$ \\
\hline & My spouse/partner abuses alcohol & 6 & $0.96 \%$ \\
\hline & Children cause care and educational problems & 26 & $4.16 \%$ \\
\hline & I have to take care of my parents & 45 & $7.20 \%$ \\
\hline & I am a one-parent family & 35 & $5.60 \%$ \\
\hline & My immediate family member suffers from long-term or severe disease & 33 & $5.28 \%$ \\
\hline & My family member is disabled & 24 & $3.84 \%$ \\
\hline \multicolumn{2}{|c|}{ Occupational and Competency Variables } & $\mathbf{N}$ & $\%$ \\
\hline \multirow[t]{7}{*}{ Net monthly income (PLN) } & $1000-2000$ & 72 & $11.52 \%$ \\
\hline & $200 I-3000$ & 348 & $55.68 \%$ \\
\hline & $300 I-4000$ & 130 & $20.80 \%$ \\
\hline & $400 I-5000$ & 37 & $5.92 \%$ \\
\hline & $500 \mathrm{I}-6000$ & 13 & $2.08 \%$ \\
\hline & $>6001$ & 5 & $0.80 \%$ \\
\hline & No answer & 20 & $3.20 \%$ \\
\hline
\end{tabular}

(Continued) 
Table I (Continued).

\begin{tabular}{|c|c|c|c|}
\hline Job tenure (years) & $\begin{array}{l}0-5 \\
6-10 \\
11-15 \\
16-20 \\
>21 \\
\text { No answer }\end{array}$ & $\begin{array}{l}126 \\
63 \\
39 \\
82 \\
301 \\
14\end{array}$ & $\begin{array}{l}20.16 \% \\
10.08 \% \\
6.24 \% \\
13.12 \% \\
48.16 \% \\
2.24 \%\end{array}$ \\
\hline Postgraduate education* & $\begin{array}{l}\text { Qualifying courses } \\
\text { Specialization } \\
\text { Specialist courses } \\
\text { Other forms }\end{array}$ & $\begin{array}{l}400 \\
163 \\
358 \\
82\end{array}$ & $\begin{array}{l}64.00 \% \\
26.08 \% \\
57.28 \% \\
13.12 \%\end{array}$ \\
\hline Number of places of work & $\begin{array}{l}1 \\
2 \\
>3 \\
\text { No answer }\end{array}$ & $\begin{array}{l}334 \\
192 \\
85 \\
20\end{array}$ & $\begin{array}{l}53.44 \% \\
30.72 \% \\
13.60 \% \\
2.77 \%\end{array}$ \\
\hline Main place of work & $\begin{array}{l}\text { Inpatient health care } \\
\text { Outpatient health care } \\
\text { Medical university } \\
\text { School, foundling hospital, children's shelter } \\
\text { Nursing home care } \\
\text { Nursery } \\
\text { Private corporation-type care } \\
\text { Long-term care } \\
\text { Antenatal classes } \\
\text { Self-government of nurses and midwives } \\
\text { Other } \\
\text { No answer }\end{array}$ & $\begin{array}{l}525 \\
49 \\
4 \\
1 \\
1 \\
3 \\
22 \\
5 \\
0 \\
0 \\
11 \\
4\end{array}$ & $\begin{array}{l}84.00 \% \\
7.84 \% \\
0.64 \% \\
0.16 \% \\
0.16 \% \\
0.48 \% \\
3.52 \% \\
0.80 \% \\
0.00 \% \\
0.00 \% \\
1.76 \% \\
0.64 \%\end{array}$ \\
\hline Time spent at work [h/month] & $\begin{array}{l}<100 \\
101-180 \\
181-230 \\
231-300 \\
>301 \\
\text { No answer }\end{array}$ & $\begin{array}{l}28 \\
386 \\
141 \\
50 \\
7 \\
13\end{array}$ & $\begin{array}{l}4.48 \% \\
61.76 \% \\
22.56 \% \\
8.00 \% \\
1.12 \% \\
2.08 \%\end{array}$ \\
\hline Amount of sleep [h/week] & $\begin{array}{l}<30 \\
31-60 \\
>6 \mathrm{I} \\
\text { No answer }\end{array}$ & $\begin{array}{l}113 \\
446 \\
36 \\
30\end{array}$ & $\begin{array}{l}18.08 \% \\
71.36 \% \\
5.76 \% \\
4.80 \%\end{array}$ \\
\hline Amount of free time $[\mathrm{h} /$ week] & $\begin{array}{l}<10 \\
1 \mathrm{I}-20 \\
>2 \mathrm{I} \\
\text { No answer }\end{array}$ & $\begin{array}{l}269 \\
249 \\
82 \\
25\end{array}$ & $\begin{array}{l}43.04 \% \\
39.84 \% \\
13.12 \% \\
4.00 \%\end{array}$ \\
\hline Nature of work & $\begin{array}{l}\text { I work independently } \\
\text { I lead a group of employees } \\
\text { I work in a group } \\
\text { No answer }\end{array}$ & $\begin{array}{l}65 \\
50 \\
498 \\
12\end{array}$ & $\begin{array}{l}10.40 \% \\
8.00 \% \\
79.68 \% \\
1.92 \%\end{array}$ \\
\hline
\end{tabular}

(Continued) 
Table I (Continued).

\begin{tabular}{|c|c|c|c|}
\hline System of work & $\begin{array}{l}\text { 8-hour shift } \\
\text { 12-hour shift } \\
\text { Other } \\
\text { No answer }\end{array}$ & $\begin{array}{l}|4| \\
403 \\
39 \\
42\end{array}$ & $\begin{array}{l}22.56 \% \\
64.48 \% \\
6.24 \% \\
6.72 \%\end{array}$ \\
\hline Number of patients & $\begin{array}{l}1-5 \\
6-15 \\
16-25 \\
26-35 \\
>36 \\
\text { No answer }\end{array}$ & $\begin{array}{l}132 \\
143 \\
118 \\
100 \\
102 \\
30\end{array}$ & $\begin{array}{l}21.12 \% \\
22.88 \% \\
18.88 \% \\
16.00 \% \\
16.32 \% \\
4.80 \%\end{array}$ \\
\hline Patients & $\begin{array}{l}\text { Pediatric patients } \\
\text { Adult patients } \\
\text { Geriatric patients } \\
\text { Oncology patients } \\
\text { Other }\end{array}$ & $\begin{array}{l}133 \\
431 \\
151 \\
142 \\
60\end{array}$ & $\begin{array}{l}21.28 \% \\
68.96 \% \\
24.16 \% \\
22.72 \% \\
9.60 \%\end{array}$ \\
\hline
\end{tabular}

Note: *Percentages do not sum to $100 \%$ because that was a multiple-choice question.

Table 2 Characteristics of the Study Group

\begin{tabular}{|c|c|c|c|c|c|}
\hline Tool & \multicolumn{3}{|c|}{ Parameter } & \multicolumn{2}{|c|}{ Result } \\
\hline \multirow[t]{5}{*}{ SWJS } & & \\
\hline & \multicolumn{2}{|l|}{ M (SD) } & \multicolumn{3}{|c|}{$17.13(7.06)$} \\
\hline & \multicolumn{3}{|l|}{ Me } & \multicolumn{2}{|c|}{18} \\
\hline & \multicolumn{3}{|l|}{ Min - max } & \multicolumn{2}{|c|}{$5-35$} \\
\hline & \multicolumn{2}{|l|}{ QI; Q3 } & \multicolumn{3}{|c|}{$10.5 ; 23$} \\
\hline \multirow[t]{8}{*}{ SWLS } & \multicolumn{3}{|l|}{$\mathrm{N}$} & \multicolumn{2}{|c|}{577} \\
\hline & \multicolumn{2}{|l|}{$M(S D)$} & \multicolumn{3}{|c|}{$19.55(7.86)$} \\
\hline & & \multicolumn{3}{|c|}{21} \\
\hline & \multicolumn{2}{|l|}{ Min - max } & \multicolumn{3}{|l|}{$5-35$} \\
\hline & \multicolumn{2}{|l|}{ QI; Q3 } & \multicolumn{3}{|c|}{$12 ; 25$} \\
\hline & \multirow{3}{*}{\multicolumn{2}{|c|}{$\begin{array}{l}\text { Low life satisfaction } \\
\text { Medium life satisfaction } \\
\text { High life satisfaction }\end{array}$}} & & 218 & $37.78 \%$ \\
\hline & & & & 147 & $25.48 \%$ \\
\hline & & & & 212 & $36.74 \%$ \\
\hline \multirow[t]{8}{*}{ LOT-R } & \multirow{5}{*}{\multicolumn{2}{|c|}{$\begin{array}{l}N \\
M(S D) \\
M e \\
\text { Min - max } \\
\text { QI; Q3 }\end{array}$}} & \multirow{5}{*}{\multicolumn{3}{|c|}{$\begin{array}{l}564 \\
14.38(3.62) \\
14 \\
1-24 \\
12 ; 17\end{array}$}} \\
\hline & & & & & \\
\hline & & & & & \\
\hline & & & & & \\
\hline & & & & & \\
\hline & A tendency & & 197 & & $34.93 \%$ \\
\hline & Neutral ori & & 211 & & $37.41 \%$ \\
\hline & A tendency & & 156 & & $27.66 \%$ \\
\hline MBI & & $\mathrm{EE}$ & DEP & PA & Total \\
\hline & $N$ & 586 & 580 & 576 & 576 \\
\hline & $M(S D)$ & $33.67(26.86)$ & $23.65(27.47)$ & 44.88 (31.99) & $34.13(18.84)$ \\
\hline & $\mathrm{Me}$ & 22.22 & 20 & 37.5 & 32.87 \\
\hline & Min - max & $0-100$ & $0-100$ & $0-100$ & $0-96.3$ \\
\hline & QI; Q3 & II.II; 55.56 & $0 ; 40$ & $12.5 ; 75$ & $20.83 ; 44.93$ \\
\hline
\end{tabular}

Abbreviations: SWJS, Satisfaction with Job Scale; SWLS, Satisfaction with Life Scale; LOT-R, Life Orientation Test - Revised version; MBI, Maslach Burnout Inventory; EE, emotional exhaustion; DEP, depersonalization; PA, personal accomplishment. 
Table 3 Correlations Between Burnout (MBI) and Job Satisfaction (SWJS)

\begin{tabular}{|l|l|l|}
\hline \multirow{2}{*}{ MBI } & SWJS & \multirow{2}{*}{ P } \\
\cline { 2 - 2 } & Spearman Correlation Coefficient & \\
\hline EE & -0.061 & 0.148 \\
DEP & 0.257 & $<0.001$ \\
PA & -0.654 & $<0.001$ \\
MBI total & -0.354 & $<0.001$ \\
\hline
\end{tabular}

Abbreviations: SWJS, Satisfaction with Job Scale; MBI, Maslach Burnout Inventory; $\mathrm{EE}$, emotional exhaustion; DEP, depersonalization; PA, personal accomplishment.

domain $(r=0.061 ; p=0.148)$. The results of selected correlations are presented in Table 3.

\section{Effects of Individual Variables on the Level of Occupational Burnout (MBI)} Emotional Exhaustion (EE)

Respondents living in poor and sufficient housing conditions were more emotionally exhausted than those living in good housing conditions $(\mathrm{p}=0.025)$. EE was significantly greater in respondents with medium life satisfaction than in those with low and high scores on the SWLS ( $p<0.001)$; EE was also significantly greater in pessimists and those with a neutral orientation than in optimists on the LOT-R scale $(\mathrm{p}=0.003)$. EE was significantly greater in respondents working in two places of work $(\mathrm{p}=0.024)$ and in those working in inpatient health care $(\mathrm{p}<0.001)$. Respondents providing care for 26-35 patients were more exhausted than those providing care for $1-5$ patients $(\mathrm{p}=0.031)$. Moreover, respondents who provided care for pediatric patients had lower EE levels $(\mathrm{p}<0.001)$, and those who provided care for geriatric patients had higher EE levels $(p=0.028)$. Those reporting less than $21 \mathrm{~h}$ of free time per week were more exhausted. The results for the effects of variables on the EE domain of MBI score are presented in Table 4.

\section{Depersonalization (DEP)}

Respondents living in poor and sufficient housing conditions and those reporting problems in the family had higher DEP levels ( $\mathrm{p}=0.01$ and $\mathrm{p}=0.009$, respectively). DEP was significantly greater in those with medium scores on the SWLS than in those with high scores. In turn, DEP in respondents with high scores on the SWLS was higher than in those with low life satisfaction $(p<0.001)$. DEP was significantly greater in respondents with a neutral orientation than in pessimists on the LOT-R scale $(p=0.015)$. DEP level depended significantly on the main place of work, its nature, and its system. DEP was significantly greater in respondents working in inpatient health care $(p<0.001)$ and those working in a group $(p=0.023)$. Shift nurses had higher DEP levels $(p=0.003)$. Furthermore, respondents who provided care for pediatric patients had lower DEP levels $(\mathrm{p}<0.045)$, and those who provided care for geriatric patients had higher DEP levels $(p=0.039)$. The effects of variables on the DEP domain of MBI score are presented in Table 5.

\section{Personal Accomplishment (PA)}

Reduced PA was significantly greater in respondents with a bachelor's degree than in those with a master's degree and graduates of a secondary medical school $(p<0.001)$. Decreased PA was significantly greater in respondents with low life satisfaction than in those with medium life satisfaction, while in those with medium scores on the SWLS, lowered PA was higher than in respondents with high scores $(\mathrm{p}<0.001)$. Reduced PA was significantly greater in pessimists than in respondents with a neutral orientation, while it was higher in neutrals than in optimists on the LOT-R scale $(p<0.001)$. The results of the post hoc analysis found that job dissatisfaction was significantly greater in those with job tenure of $6-10$ years $(\mathrm{p}=0.043)$. Respondents who did not take qualifying $(\mathrm{p}=0.001)$ and specialist $(\mathrm{p}=0.016)$ courses reported more decreased PA. Reduced PA was also higher in those who worked only in one place of work $(\mathrm{p}=0.023)$. Respondents working primarily in inpatient health care had more decreased PA $(p=0.003)$. Group leaders had significantly lower job dissatisfaction $(\mathrm{p}<0.001)$, while $12 \mathrm{~h}$-shift nurses had higher scores indicating lowered PA ( $\mathrm{p}=0.028)$. Reduced PA was significantly greater in respondents with less than 10 hours of free time per week. The effects of variables on lowered PA domain of MBI score are presented in Table 6.

\section{Total MBI}

The results found that occupational burnout was significantly higher in respondents with low and medium life satisfaction as well as in pessimists and neutrals (both $\mathrm{p}<0.001)$. Total MBI depended significantly on the main place of work, its nature, and its system. The results of the post hoc analysis found that total MBI was significantly higher in those who worked primarily in inpatient health care $(p<0.001)$. Group leaders and independent nurses showed a significantly lower total MBI level $(p=0.04)$. Shift nurses had higher scores on the total MBI $(p=0.005)$. Respondents providing care for pediatric 
Table 4 The effects of examined variables on burnout level - emotional exhaustion (EE)

\begin{tabular}{|c|c|c|c|c|c|c|c|c|}
\hline \multicolumn{2}{|c|}{ Sociodemographic Variables } & \multirow{3}{*}{$\begin{array}{l}\mathbf{N} \\
494 \\
89\end{array}$} & \multirow{3}{*}{$\begin{array}{l}\mathbf{M} \\
32.87 \\
38.16\end{array}$} & \multirow{3}{*}{$\begin{array}{l}\text { SD } \\
27.18 \\
24.7\end{array}$} & \multirow{3}{*}{$\begin{array}{l}\text { Me } \\
22.22 \\
33.33\end{array}$} & \multirow{3}{*}{$\begin{array}{l}\text { QI } \\
11.11 \\
22.22\end{array}$} & \multirow{3}{*}{$\begin{array}{l}\text { Q3 } \\
55.56 \\
55.56\end{array}$} & \multirow{3}{*}{$\begin{array}{l}\mathbf{p} \\
0.025\end{array}$} \\
\hline Housing conditions ${ }^{b}$ & Good & & & & & & & \\
\hline & Poor, sufficient & & & & & & & \\
\hline \multicolumn{2}{|c|}{ Health-Related and Psychological Variables } & $\mathbf{N}$ & $\mathbf{M}$ & SD & Me & QI & Q3 & $\mathbf{p}$ \\
\hline \multirow[t]{3}{*}{$\mathrm{SWLS}^{\mathrm{a}}$} & Low (L) & 214 & 31.25 & 26.03 & 22.22 & 11.11 & 44.44 & $<0.001$ \\
\hline & Medium (M) & 143 & 41.4 & 27.78 & 33.33 & 22.22 & 62.5 & $M>L, H$ \\
\hline & High $(H)$ & 201 & 29.5 & 25.21 & 22.22 & 11.11 & 44.44 & \\
\hline \multirow[t]{3}{*}{ LOT-R $^{\mathrm{a}}$} & Pessimism (P) & 192 & 35.66 & 26.63 & 25 & 11.11 & 55.56 & 0.003 \\
\hline & Neutral $(\mathrm{N})$ & 209 & 35.3 & 27.64 & 25 & 11.11 & 55.56 & $\mathrm{PN}>\mathrm{O}$ \\
\hline & Optimism (O) & 150 & 26.75 & 23.89 & 22.22 & 11.11 & 42.71 & \\
\hline \multicolumn{2}{|c|}{ Occupational and Competency Variables } & $\mathbf{N}$ & $\mathbf{M}$ & SD & Me & QI & Q3 & $\mathbf{p}$ \\
\hline \multirow[t]{3}{*}{ Number of places of work ${ }^{a}$} & 1 & 315 & 31.36 & 26.67 & 22.22 & 11.11 & 44.44 & 0.024 \\
\hline & 2 & $|8|$ & 37.17 & 26.65 & 33.33 & 22.22 & 55.56 & $2>1$ \\
\hline & $>3$ & 79 & 33.91 & 25.99 & 25 & 11.11 & 55.56 & \\
\hline \multirow[t]{2}{*}{ Main places of work ${ }^{b}$} & Inpatient health care & 493 & 35.17 & 26.78 & 25 & 11.11 & 55.56 & $<0.001$ \\
\hline & Other & 93 & 25.73 & 25.98 & 22.22 & 11.11 & 37.5 & \\
\hline \multirow[t]{5}{*}{ Number of patients ${ }^{\mathrm{a}}$} & $1-5$ & 120 & 27.31 & 22.62 & 22.22 & 11.11 & 44.44 & 0.031 \\
\hline & $6-15$ & 136 & 35.69 & 26.67 & 25 & 12.15 & 55.56 & $26-35>1-5$ \\
\hline & $16-25$ & 116 & 36.07 & 27.61 & 25 & 11.11 & 55.56 & \\
\hline & $26-35$ & 93 & 38.81 & 29.89 & 33.33 & 12.5 & 62.5 & \\
\hline & $>35$ & 96 & 32.31 & 27.36 & 22.22 & 11.11 & 51.39 & \\
\hline \multirow[t]{2}{*}{ Paediatric patients ${ }^{b}$} & No & 462 & 35.61 & 26.95 & 25 & 11.11 & 55.56 & $<0.001$ \\
\hline & Yes & 124 & 26.42 & 25.34 & 22.22 & 11.11 & 44.44 & \\
\hline \multirow[t]{2}{*}{ Geriatric patients $^{\mathrm{b}}$} & No & 447 & 32.17 & 25.95 & 22.22 & 11.11 & 47.22 & 0.028 \\
\hline & Yes & 139 & 38.49 & 29.16 & 33.33 & 12.5 & 55.56 & \\
\hline \multirow[t]{3}{*}{ Free time $[\mathrm{h} / \text { week }]^{\mathrm{a}}$} & $<10(A)$ & 254 & 35.44 & 26.77 & 25 & 11.11 & 55.56 & 0.008 \\
\hline & $\mathrm{II}-20$ (B) & 235 & 34.82 & 27.76 & 22.22 & 11.11 & 55.56 & $A B>C$ \\
\hline & $>2 \mathrm{lh}(\mathrm{C})$ & 76 & 25.16 & 23.29 & 22.22 & 11.11 & 33.33 & \\
\hline
\end{tabular}

Notes: ${ }^{a}$ Kruskal-Wallis test and post-hoc analysis (Dunn's test), ' $M a n n-W h i t n e y ~ U$-test.

Abbreviations: LOT-R, Life Orientation Test-Revised version; EE, emotional exhaustion.

patients had significantly lower MBI levels $(\mathrm{p}<0.001)$. Total MBI was significantly greater in respondents with less than $20 \mathrm{~h}$ of free time per week. The results for the effects of variables on the total MBI score are presented in Table 7.

\section{Predictors of Occupational Burnout - Multiple Regression Analysis \\ Emotional Exhaustion (EE)}

The linear regression model found that independent predictors of the burnout level in the domain of EE included (1) the main place of work (other places of work lower the burnout level by 7.993 pts on average; $p=0.023$ ); (2) average number of patients under care (6-15 patients elevate the burnout level by 7.868 pts on average; $\mathrm{p}=0.017$ and $26-35$ patients elevate the burnout level by 7.452 pts on average; $p=0.046$ ); (3) life satisfaction (medium life satisfaction elevates the burnout level by 13.626 pts on average; $p<0.001$, while high life satisfaction elevates the burnout level by $8.285 \mathrm{pts}$ on average; $\mathrm{p}=0.004$ ). The $\mathrm{R}^{2}$ determination coefficient of the EE domain was $26.54 \%$, which means that the remaining $73.46 \%$ depended on variables not included in the model and random factors. The predictors of burnout for the EE domain are presented in Table 8.

\section{Depersonalization (DEP)}

The linear regression model showed that independent predictors of the burnout level in the domain of DEP included 
Table 5 The effects of examined variables on burnout level - depersonalization (DEP)

\begin{tabular}{|c|c|c|c|c|c|c|c|c|}
\hline \multicolumn{2}{|c|}{ Sociodemographic Variables } & \multirow{3}{*}{$\begin{array}{l}\mathbf{N} \\
488 \\
89\end{array}$} & \multirow{3}{*}{$\begin{array}{l}\mathbf{M} \\
22.32 \\
31.52\end{array}$} & \multirow{3}{*}{$\begin{array}{l}\text { SD } \\
26.49 \\
31.55\end{array}$} & \multirow{3}{*}{$\begin{array}{l}\text { Me } \\
20 \\
20\end{array}$} & \multirow{3}{*}{$\begin{array}{l}\text { QI } \\
0 \\
0\end{array}$} & \multirow{3}{*}{$\begin{array}{l}\text { Q3 } \\
40 \\
50\end{array}$} & \multirow{3}{*}{$\begin{array}{l}\mathbf{p} \\
0.01\end{array}$} \\
\hline Housing conditions ${ }^{\mathrm{b}}$ & Good & & & & & & & \\
\hline & Poor, sufficient & & & & & & & \\
\hline \multirow[t]{2}{*}{ Family-related problems ${ }^{b}$} & No & 437 & 21.99 & 26.61 & 20 & 0 & 40 & 0.009 \\
\hline & Yes & 143 & 28.71 & 29.45 & 20 & 0 & 40 & \\
\hline \multicolumn{2}{|c|}{ Health-Related and Psychological Variables } & $\mathbf{N}$ & M & SD & Me & QI & Q3 & $\mathbf{p}$ \\
\hline \multirow[t]{3}{*}{ SWLS ${ }^{a}$} & Low (L) & 214 & 15.19 & 23.87 & 0 & 0 & 20 & $<0.001$ \\
\hline & Medium (M) & $|4|$ & 32.52 & 28.94 & 20 & 20 & 40 & $M>H>L$ \\
\hline & High $(H)$ & 198 & 26.09 & 27.32 & 20 & 0 & 40 & \\
\hline \multirow[t]{3}{*}{ LOT-R $^{\mathrm{a}}$} & Pessimism (P) & 192 & 20.03 & 26.99 & 0 & 0 & 40 & 0.015 \\
\hline & Neutral $(\mathrm{N})$ & 204 & 26.84 & 28.06 & 20 & 0 & 40 & $N>P$ \\
\hline & Optimism (O) & 150 & 24 & 27.21 & 20 & 0 & 40 & \\
\hline \multicolumn{2}{|c|}{ Occupational and Competency Variables } & $\mathbf{N}$ & M & SD & Me & QI & Q3 & $\mathbf{p}$ \\
\hline \multirow[t]{2}{*}{ Main places of work ${ }^{b}$} & Inpatient health care & 488 & 25.32 & 27.59 & 20 & 0 & 40 & $<0.001$ \\
\hline & Other & 92 & 14.78 & 25.17 & 0 & 0 & 20 & \\
\hline \multirow[t]{3}{*}{ Nature of work ${ }^{\mathrm{a}}$} & I work independently (A) & 61 & 15.74 & 24.25 & 0 & 0 & 20 & 0.023 \\
\hline & I lead a group of employees (B) & 48 & 23.85 & 28.4 & 20 & 0 & 40 & $C>A$ \\
\hline & I work in a group $(\mathrm{C})$ & 461 & 24.6 & 27.56 & 20 & 0 & 40 & \\
\hline \multirow[t]{3}{*}{ System of work ${ }^{a}$} & 8-hour shift (8) & 129 & 18.53 & 24.99 & 0 & 0 & 25 & 0.003 \\
\hline & 12-hour shift (12) & 379 & 26.11 & 28.33 & 20 & 0 & 40 & $12>8,0$ \\
\hline & Other $(O)$ & 36 & 15.56 & 23.84 & 0 & 0 & 20 & \\
\hline \multirow[t]{2}{*}{ Geriatric patients $^{\mathrm{b}}$} & No & 443 & 22.04 & 26 & 20 & 0 & 40 & 0.039 \\
\hline & Yes & 137 & 28.83 & 31.31 & 20 & 0 & 40 & \\
\hline \multirow[t]{2}{*}{ Pediatric patients ${ }^{\mathrm{b}}$} & No & 458 & 25.11 & 28.57 & 20 & 0 & 40 & 0.045 \\
\hline & Yes & 122 & 18.16 & 22.14 & 20 & 0 & 40 & \\
\hline
\end{tabular}

Notes: ${ }^{a}$ Kruskal-Wallis test and post-hoc analysis (Dunn's test), 'Mann-Whitney U-test.

Abbreviations: SWLS, Satisfaction with Life Scale; LOT-R, Life Orientation Test-Revised version; DEP, depersonalization.

(1) job satisfaction (each additional score on the SWJS questionnaire elevates the burnout level by $0.837 \mathrm{pts}$ on average; $p<0.001$ ); (2) family-related problems (they elevate the burnout level by $5.788 \mathrm{pts}$ on average; $\mathrm{p}=0.041$ ); (3) level of life satisfaction (medium life satisfaction elevates the burnout level by 13.296 pts on average; $\mathrm{p}<0.001)$. The $\mathrm{R}^{2}$ determination coefficient of the DEP domain was $21.91 \%$, which means that the remaining $78.09 \%$ depended on variables not included in the model and random factors. The predictors of burnout for the DEP domain are presented in Table 8 .

\section{Personal Accomplishment (PA)}

The linear regression model showed that independent predictors of the burnout level in the domain of reduced PA included (1) job satisfaction (each additional score on the
SWJS questionnaire lowers the burnout level by $1.974 \mathrm{pts}$ on average; $\mathrm{p}<0.01$ ); (2) job tenure (compared to job tenure of $0-5$ years, job tenure $>20$ years lowers the burnout level by 7.633 pts on average; $\mathrm{p}=0.016$ ); (3) level of life satisfaction (medium life satisfaction lowers the burnout level by 12.866 pts on average; $p<0.001$, and high life satisfaction lowers the burnout level by 16.175 pts on average; $\mathrm{p}<0.001$ ); (4) life orientation (neutral orientation lowers the burnout level by $6.45 \mathrm{pts}$ on average; $\mathrm{p}=0.014$ and optimism lowers the burnout level by 10.351 pts on average; $\mathrm{p}=0.001$ ). The $\mathrm{R}^{2}$ determination coefficient of the PA domain was $56.96 \%$, which means that the remaining $43.04 \%$ depended on variables not included in the model and random factors. The predictors of burnout for the PA domain are presented in Table 8 . 
Table 6 The effects of examined variables on burnout level - personal accomplishment (PA)

\begin{tabular}{|c|c|c|c|c|c|c|c|c|}
\hline \multicolumn{2}{|c|}{ Sociodemographic Variables } & \multirow{2}{*}{$\begin{array}{l}\mathbf{N} \\
120\end{array}$} & \multirow{2}{*}{$\begin{array}{l}M \\
49.66\end{array}$} & \multirow{2}{*}{$\begin{array}{l}\text { SD } \\
31.23\end{array}$} & \multirow{2}{*}{$\begin{array}{l}\text { Me } \\
50\end{array}$} & \multirow{2}{*}{$\begin{array}{l}\text { Q I } \\
25\end{array}$} & \multirow{2}{*}{$\begin{array}{c}\text { Q3 } \\
75\end{array}$} & \multirow{3}{*}{$\begin{array}{l}\mathbf{p} \\
0.043 \\
6-10>16-20\end{array}$} \\
\hline Job tenure (years) ${ }^{a}$ & $0-5$ & & & & & & & \\
\hline & $6-10$ & 58 & 50.49 & 28.35 & 50 & 25 & 75 & \\
\hline & $11-15$ & 34 & 49.89 & 33.49 & 50 & 15.62 & 87.5 & \\
\hline & $16-20$ & 78 & 39.55 & 32.32 & 37.5 & 12.5 & 62.5 & \\
\hline & $>21$ & 274 & 42.4 & 32.52 & 37.5 & 12.5 & 69.2 & \\
\hline \multirow[t]{4}{*}{ Education $^{\mathrm{a}}$} & Secondary medical school (SMS) & 121 & 40.53 & 31.72 & 37.5 & 12.5 & 62.5 & $<0.001$ \\
\hline & Medical vocational school (MVS) & 37 & 41.8 & 32.79 & 37.5 & 12.5 & 62.5 & $B A>S M S, M A$ \\
\hline & Bachelor's degree (BA) & 261 & 51.97 & 32.07 & 50 & 25 & 85.71 & \\
\hline & Master's degree (MA) & 146 & 37.1 & 29.68 & 25 & 12.5 & 62.5 & \\
\hline \multicolumn{2}{|c|}{ Health-Related and Psychological Variables } & $\mathbf{N}$ & $\mathbf{M}$ & SD & Me & QI & Q3 & $\mathbf{p}$ \\
\hline \multirow[t]{3}{*}{$S W L S^{a}$} & Low (L) & 210 & 68.07 & 28.35 & 75 & 50 & 87.5 & $<0.001$ \\
\hline & Medium (M) & $|4|$ & 37.71 & 24.92 & 37.5 & 16.67 & 50 & $\mathrm{~L}>\mathrm{M}>\mathrm{H}$ \\
\hline & High $(H)$ & 198 & 26.44 & 24.16 & 25 & 12.5 & 37.5 & \\
\hline \multirow[t]{3}{*}{ LOT-R $^{\mathrm{a}}$} & Pessimism (P) & 190 & 61.9 & 29.15 & 62.5 & 37.5 & 87.5 & $<0.001$ \\
\hline & Neutral $(\mathrm{N})$ & 202 & 44.06 & 31.45 & 37.5 & 12.95 & 75 & $\mathrm{P}>\mathrm{N}>\mathrm{O}$ \\
\hline & Optimism (O) & 150 & 26.4 & 24.14 & 25 & 0 & 37.5 & \\
\hline \multicolumn{2}{|c|}{ Occupational and Competency Variables } & $\mathbf{N}$ & $\mathbf{M}$ & SD & Me & QI & Q3 & $\mathbf{p}$ \\
\hline \multirow[t]{2}{*}{ Qualifying courses $^{\mathrm{b}}$} & No courses & 206 & 50.67 & 31.34 & 50 & 25 & 75 & 0.001 \\
\hline & Qualifying courses & 370 & 41.66 & 31.93 & 37.5 & 12.5 & 62.5 & \\
\hline \multirow[t]{2}{*}{ Specialist courses ${ }^{\mathrm{b}}$} & No courses & 240 & 48.68 & 32.57 & 50 & 25 & 75 & 0.016 \\
\hline & Specialist courses & 336 & 42.17 & 31.33 & 37.5 & 12.5 & 62.5 & \\
\hline \multirow[t]{3}{*}{ Number of places of work ${ }^{a}$} & 1 & 310 & 47.69 & 31.9 & 50 & 25 & 75 & 0.023 \\
\hline & 2 & 177 & 42.7 & 31.79 & 37.5 & 12.5 & 62.5 & $1>3+$ \\
\hline & $3+$ & 78 & 37.39 & 30.95 & 37.5 & 12.5 & 62.5 & \\
\hline \multirow[t]{2}{*}{ Main places of work } & Inpatient health care & 486 & 46.51 & 31.63 & 50 & 25 & 75 & 0.003 \\
\hline & Other & 90 & 36.06 & 32.64 & 25 & 12.5 & 62.5 & \\
\hline \multirow[t]{3}{*}{ Nature of work ${ }^{a}$} & I work independently (A) & 59 & 43.17 & 30.48 & 37.5 & 20.83 & 62.5 & $<0.001$ \\
\hline & I lead a group of employees (B) & 48 & 25.76 & 27.34 & 20.83 & 0 & 37.5 & $A C>B$ \\
\hline & I work in a group (C) & 459 & 47.48 & 31.86 & 50 & 25 & 75 & \\
\hline \multirow[t]{3}{*}{ System of work ${ }^{\mathrm{a}}$} & 8-hour shift (8) & 128 & 39.45 & 31.94 & 37.5 & 12.5 & 62.5 & 0.028 \\
\hline & I2-hour shift (I2) & 377 & 47.6 & 31.31 & 50 & 25 & 75 & $12>8,0$ \\
\hline & Other $(\mathrm{O})$ & 36 & 42.16 & 34.99 & 31.25 & 12.5 & 75 & \\
\hline \multirow[t]{3}{*}{ Free time $[\mathrm{h} / \text { week }]^{\mathrm{a}}$} & $<10(A)$ & 251 & 49.11 & 32.69 & 50 & 25 & 75 & 0.006 \\
\hline & $\mathrm{II}-20(\mathrm{~B})$ & 228 & 42.87 & 30.72 & 37.5 & 14.29 & 62.5 & $A>C$ \\
\hline & $>2 \operatorname{lh}(\mathrm{C})$ & 76 & 36.72 & 32.22 & 25 & 12.5 & 62.5 & \\
\hline
\end{tabular}

Notes: ${ }^{2}$ Kruskal-Wallis test and post-hoc analysis (Dunn's test), ${ }^{b}$ Mann-Whitney U-test.

Abbreviations: SWLS, Satisfaction with Life Scale; LOT-R, Life Orientation Test-Revised version; PA, personal accomplishment.

\section{Total MBI}

The linear regression model showed that independent predictors of the burnout level in the domain of total MBI included (1) main place of work (other places of work lower the burnout level by $6.029 \mathrm{pts}$ on average; $\mathrm{p}=0.026$ ); and (2) life orientation (optimism lowers the burnout level by 7.039 pts on average; $\mathrm{p}=0.001$ ). The $\mathrm{R}^{2}$ determination coefficient of the total MBI score was $32.22 \%$, which means that the remaining $67.78 \%$ depended on variables not included in the model and random factors. The 
Table 7 The Effects of Examined Variables on Burnout Level - Total MBI

\begin{tabular}{|c|c|c|c|c|c|c|c|c|}
\hline \multicolumn{2}{|c|}{ Health-Related and Psychological Variables } & \multirow{4}{*}{$\begin{array}{l}\mathbf{N} \\
210 \\
141 \\
198\end{array}$} & \multirow{4}{*}{$\begin{array}{l}M \\
38.37 \\
37.17 \\
27.22\end{array}$} & \multirow{4}{*}{$\begin{array}{l}\text { SD } \\
15.77 \\
19.47 \\
19.02\end{array}$} & \multirow{4}{*}{$\begin{array}{l}\text { Me } \\
36.57 \\
35 \\
24.21\end{array}$} & \multirow{4}{*}{$\begin{array}{l}\text { QI } \\
28.7 \\
21.67 \\
13.06\end{array}$} & \multirow{4}{*}{$\begin{array}{l}\text { Q3 } \\
44.05 \\
52.69 \\
38.03\end{array}$} & \multirow{4}{*}{$\begin{array}{l}\mathbf{p} \\
<0.00 \mathrm{I} \\
\mathrm{L}, \mathrm{M}>\mathrm{H}\end{array}$} \\
\hline SWLS $^{\mathrm{a}}$ & Low (L) & & & & & & & \\
\hline & Medium (M) & & & & & & & \\
\hline & High $(H)$ & & & & & & & \\
\hline \multirow[t]{3}{*}{ LOT-R ${ }^{a}$} & Pessimism (P) & 190 & 39.35 & 17 & 36.57 & 28.24 & 48.15 & $<0.001$ \\
\hline & Neutral (N) & 202 & 35.41 & 17.96 & 35.65 & 23.19 & 46.83 & $\mathrm{PN}>\mathrm{O}$ \\
\hline & Optimism (O) & 150 & 25.72 & 19.08 & 22.33 & 12.15 & 33.98 & \\
\hline \multicolumn{2}{|c|}{ Occupational and Competency Variables } & $\mathbf{N}$ & $\mathbf{M}$ & SD & Me & QI & Q3 & $\mathbf{p}$ \\
\hline \multirow[t]{2}{*}{ Main places of work ${ }^{b}$} & Inpatient health care & 486 & 35.67 & 18.67 & 33.84 & 22.44 & 47.73 & $<0.001$ \\
\hline & Other & 90 & 25.8 & 17.61 & 24.54 & 13.06 & 36.57 & \\
\hline \multirow[t]{3}{*}{ Nature of work ${ }^{a}$} & I work independently (A) & 59 & 29.57 & 18 & 26.85 & 18.47 & 39.63 & 0.004 \\
\hline & I lead a group of employees (B) & 48 & 27.78 & 19.8 & 26.32 & 12.38 & 37.22 & $C>A B$ \\
\hline & I work in a group $(\mathrm{C})$ & 459 & 35.47 & 18.63 & 33.33 & 22.47 & 47.08 & \\
\hline \multirow[t]{3}{*}{ System of work ${ }^{a}$} & 8-hour shift (8) & 128 & 30.03 & 19.45 & 29.26 & 15.28 & 40.74 & 0.005 \\
\hline & 12-hour shift (12) & 377 & 36.22 & 18.69 & 33.33 & 23.33 & 48.15 & $12>8,0$ \\
\hline & Other $(\mathrm{O})$ & 36 & 29.39 & 17.8 & 30.88 & 15.21 & 39 & \\
\hline \multirow[t]{2}{*}{ Paediatric patients ${ }^{b}$} & No & 457 & 35.51 & 18.88 & 34.44 & 22.41 & 46.94 & $<0.001$ \\
\hline & Yes & 119 & 28.86 & 17.77 & 28.24 & 16.53 & 37.04 & \\
\hline \multirow[t]{3}{*}{ Free time $[\mathrm{h} / \text { week }]^{\mathrm{a}}$} & $<10(\mathrm{~A})$ & 251 & 35.9 & 17.82 & 35.56 & 23.47 & 47.13 & 0.002 \\
\hline & $\mathrm{II}-20$ (B) & 228 & 34.57 & 19.2 & 32.41 & 22.41 & 45.25 & $A B>C$ \\
\hline & $>21(\mathrm{C})$ & 76 & 27.73 & 20.44 & 23.47 & 10.83 & 37.48 & \\
\hline
\end{tabular}

Notes: ${ }^{a}$ Kruskal-Wallis test and post-hoc analysis (Dunn's test), ' Mann-Whitney U-test.

Abbreviations: SWLS, Satisfaction with Life Scale; LOT-R, Life Orientation Test-Revised version; MBI, Maslach Burnout Inventory.

predictors of burnout for the total MBI score are presented in Table 8 .

\section{Discussion}

The presented study showed a higher burnout level in terms of total MBI among nurses. The most significant occupational burnout was found in the PA domain, followed by the EE domain; the fewest respondents showed burnout based on the DEP domain. Such results are consistent with studies conducted by other authors. A study by Pawlik et $\mathrm{al}^{31}$ conducted among Polish and Norwegian nurses found that as many as $75 \%$ of Polish respondents reported a low and very low level of job satisfaction, while only $16 \%$ of Norwegian nurses reported a low and very low level of job satisfaction. Also, Wilczek-Rużyczka's analysis ${ }^{32}$ proved the occurrence of EE in $20.58 \%$ of individuals, DEP in $7.29 \%$, and loss of PA in $30.84 \%$.

In the present study, studied nurses presented a moderate level of life satisfaction, including $37.78 \%$ nurses with low, $25.48 \%$ with medium, and $36.74 \%$ with high life satisfaction (SWLS); slightly lowered level of job satisfaction with a mean score of 17.13 pts. (SWJS) as well as moderate dispositional optimism including $34.93 \%$ nurses with pessimistic, 37.41 with neutral and 27.66 with optimistic life orientation (LOT-R). A study by Roczniewska and Bakker ${ }^{33}$ has shown that chronic burnout among nurses disrupts daily behavioral regulation. Individuals with elevated burnout symptoms have difficulty translating transient increases in regulatory resources into adaptive strategies associated with higher performance. The authors indicated that even burnout at mild levels disrupts daily behavioral control among nurses who are unable to capitalize on daily growth in their behavioral regulation capacity. Disturbed behavioral regulation in everyday practice can affect life orientation and attitudes toward both life and work. On the other hand, Bakker, ${ }^{34}$ based on the JD-R model, described the job crafting phenomenon as "the physical and cognitive changes individuals make in the task or relational boundaries of their work". It was shown that job crafting in the form of job resource enhancement is positively related to career development opportunities, performance feedback, and person-organization fit, especially when job engagement is high. 
Table 8 Results of the Multiple Regression Analysis

\begin{tabular}{|c|c|c|c|c|c|}
\hline \multicolumn{2}{|l|}{ Variable (EE) } & \multirow{2}{*}{$\begin{array}{l}\text { Regression Parameter } \\
\text { Ref. } \\
-7.993\end{array}$} & \multicolumn{2}{|l|}{$95 \% \mathrm{Cl}$} & \multirow{2}{*}{$\begin{array}{l}\mathbf{p} \\
0.023\end{array}$} \\
\hline Main place of work & $\begin{array}{l}\text { Inpatient health care } \\
\text { Other }\end{array}$ & & -14.873 & -1.112 & \\
\hline Average number of patients under care & $\begin{array}{l}1-5 \\
6-15 \\
16-25 \\
26-35 \\
>36\end{array}$ & $\begin{array}{l}\text { Ref. } \\
7.868 \\
1.724 \\
7.452 \\
4.649\end{array}$ & $\begin{array}{l}1.435 \\
-5.078 \\
0.138 \\
-2.737\end{array}$ & $\begin{array}{l}|4.30| \\
8.526 \\
14.765 \\
12.035\end{array}$ & $\begin{array}{l}0.017 \\
0.619 \\
0.046 \\
0.217\end{array}$ \\
\hline SWLS & $\begin{array}{l}\text { Low } \\
\text { Medium } \\
\text { High }\end{array}$ & $\begin{array}{l}\text { Ref. } \\
13.626 \\
8.285\end{array}$ & $\begin{array}{l}7.895 \\
2.604\end{array}$ & $\begin{array}{l}19.357 \\
13.967\end{array}$ & $\begin{array}{l}<0.001 \\
0.004\end{array}$ \\
\hline \multicolumn{2}{|l|}{ Variable (DEP) } & Regression Parameter & \multicolumn{2}{|l|}{$95 \% \mathrm{Cl}$} & $\mathbf{p}$ \\
\hline \multicolumn{2}{|l|}{ SWJS [pts] } & 0.837 & 0.379 & 1.295 & $<0.001$ \\
\hline Family-related problems & $\begin{array}{l}\text { No problems } \\
\text { Family-related problems }\end{array}$ & $\begin{array}{l}\text { Ref. } \\
5.788\end{array}$ & 0.227 & 11.349 & 0.041 \\
\hline SWLS & $\begin{array}{l}\text { Low } \\
\text { Medium } \\
\text { High }\end{array}$ & $\begin{array}{l}\text { Ref. } \\
13.296 \\
5.889\end{array}$ & $\begin{array}{l}6.544 \\
-1.378\end{array}$ & $\begin{array}{l}20.049 \\
13.156\end{array}$ & $\begin{array}{l}<0.001 \\
0.112\end{array}$ \\
\hline \multicolumn{2}{|l|}{ Variable (PA) } & Regression Parameter & \multicolumn{2}{|l|}{$95 \% \mathrm{Cl}$} & $\mathbf{p}$ \\
\hline \multicolumn{2}{|l|}{ SWJS [pts] } & -1.974 & -2.409 & -1.54 & $<0.001$ \\
\hline Job tenure (years) & $\begin{array}{l}0-5 \\
6-10 \\
11-15 \\
16-20 \\
>21\end{array}$ & $\begin{array}{l}\text { Ref. } \\
-1.783 \\
-0.188 \\
-6.346 \\
-7.633\end{array}$ & $\begin{array}{l}-9.472 \\
-10.237 \\
-14.215 \\
-13.825\end{array}$ & $\begin{array}{l}5.907 \\
9.86 \\
1.524 \\
-1.442\end{array}$ & $\begin{array}{l}0.649 \\
0.971 \\
0.114 \\
0.016\end{array}$ \\
\hline SWLS & $\begin{array}{l}\text { Low } \\
\text { Medium } \\
\text { High }\end{array}$ & $\begin{array}{l}\text { Ref. } \\
-12.866 \\
-16.175\end{array}$ & $\begin{array}{l}-19.137 \\
-22.915\end{array}$ & $\begin{array}{l}-6.595 \\
-9.434\end{array}$ & $\begin{array}{l}<0.001 \\
<0.001\end{array}$ \\
\hline LOT-R & $\begin{array}{l}\text { A tendency to pessimism } \\
\text { Neutral orientation } \\
\text { A tendency to optimism }\end{array}$ & $\begin{array}{l}\text { Ref. } \\
-6.45 \\
-10.35 \mid\end{array}$ & $\begin{array}{l}-11.606 \\
-16.289\end{array}$ & $\begin{array}{l}-1.295 \\
-4.413\end{array}$ & $\begin{array}{l}0.014 \\
0.001\end{array}$ \\
\hline \multicolumn{2}{|l|}{ Variable (Total MBI) } & Regression Parameter & \multicolumn{2}{|l|}{$95 \% \mathrm{Cl}$} & $\mathbf{p}$ \\
\hline Main place of work & $\begin{array}{l}\text { Inpatient health care } \\
\text { Other }\end{array}$ & $\begin{array}{l}\text { Ref. } \\
-6.029\end{array}$ & -11.322 & -0.737 & 0.026 \\
\hline LOT-R & $\begin{array}{l}\text { A tendency to pessimism } \\
\text { Neutral orientation } \\
\text { A tendency to optimism }\end{array}$ & $\begin{array}{l}\text { Ref. } \\
-1.007 \\
-7.039\end{array}$ & $\begin{array}{l}-4.68 \\
-11.279\end{array}$ & $\begin{array}{l}2.665 \\
-2.799\end{array}$ & $\begin{array}{l}0.59 \\
0.001\end{array}$ \\
\hline
\end{tabular}

Abbreviations: SWJS, Satisfaction with Job Scale; SWLS, Satisfaction with Life Scale; LOT-R, Life Orientation Test-Revised version; MBI, Maslach Burnout Inventory; EE, emotional exhaustion; DEP, depersonalization; PA, personal accomplishment.

The results obtained in the study confirm a disturbing phenomenon related to the increasing aging of medical personnel, including nurses. The majority of the study group was aged between $41-50$ years (mean age was
49.47 years). The results of this study confirm the predictions concerning the increase in the mean age of nurses, which was 44.19 years in 2008 and 50.79 years in $2016 .^{35}$ Currently, nursing is a profession that is aging at a rapid 
rate. There is a declining interest in nursing professions among young men and women. ${ }^{36}$ According to Marć et al ${ }^{37}$ the nursing shortage has reached a critical point for health care services both locally and globally. Nevertheless, in the analyzed group of nurses, age did not affect occupational burnout among nurses. No effect of age on occupational burnout was also reported by Tarcan et al. ${ }^{38}$ In contrast, Zhu et $\mathrm{al}^{39}$ observed that occupational burnout might increase with age. Some articles prove that a high level of education affects the burnout level. Better educated people are more likely to have greater expectations towards themselves, and they are more likely to hold more responsible and demanding positions. ${ }^{40}$

Nevertheless, it should be emphasized that this phenomenon may occur in nurses without such professional experience. For example, a recent cohort study by Rudman et $\mathrm{al}^{41}$ on a group of 2225 nurses revealed high levels of burnout symptoms at an early stage of their careers, including the first three years of work. It was shown that the prevalence of high levels of burnout symptoms in nurses was $12.3 \%$. In addition, burnout was significantly associated with a higher prevalence of symptoms of cognitive dysfunction (11.4\%), depression (5.7\%), and sleep disorders $(42.2 \%)$.

Most surveyed nurses earned a university degree (bachelor's and master's degrees) in the presented study. The study found that job dissatisfaction among nurses in the MBI domain was significantly higher among those with a bachelor's degree than those with a master's degree or, interestingly, graduates of a secondary medical school. In contrast, in the study by Nowak-Starz et $\mathrm{al}^{42}$ more educated nurses working in medical treatment wards and surgical wards, the highest level of occupational burnout. Nurses with a bachelor's degree were more likely to experience occupational burnout than nurses with secondary education, as confirmed by this study.

Shift work may decrease quality of life, both on a personal and professional level. Irregular working hours and 12-hour work time are predisposing factors for the development of occupational burnout. ${ }^{43}$ Dall'Ora et $\mathrm{al}^{44}$ reports that shift work for 12 or more hours should be challenged because it involves limited educational activities and reduces the opportunity to discuss patient care, which may have a negative impact and compromise the quality and safety of patient care. In that study, the results indicate that depersonalization was significantly greater in shift nurses. Furthermore, shift nurses were dissatisfied with their job and had a higher level of occupational burnout.
Therefore, shift work, especially 12-hour work time, is considered a stressor. ${ }^{45}$ Job tenure was a factor affecting occupational burnout in the domain of job dissatisfaction. Job dissatisfaction was significantly greater among nurses with job tenure of 6-10 years. It was higher than in those working more than 16 years in the nursing profession.

Research concerning the impact of job tenure of nurses on occupational burnout remains inconclusive. Kędra and Sanak $^{46}$ and Marcysiak et $\mathrm{al}^{47}$ indicate that the greater the job tenure of nurses, the greater their occupational burnout. Several other studies prove a similar relationship. Furthermore, Haor et $\mathrm{al}^{48}$ suggest that job tenure decreases positive attitudes towards work and increases life satisfaction. In contrast, another study by Al-Turki et $\mathrm{al}^{49}$ that analyses the effect of job tenure shows that the greater the job tenure, the lower the occupational burnout, which reflects the results of our study.

In the study by Sodomska et $\mathrm{al}^{50}$ nurses with longer job tenure had a lower level of depersonalization. Differences in the reports concerning the effects of job tenure on the level of occupational burnout may be due to using more or less effective ways of coping with stress, the level of social competencies, as well as having individual personal traits that may even predispose to the development of occupational burnout. ${ }^{17,51,52}$

\section{Study Limitations}

This study has some potential limitations to be mentioned. First of all, the group of nurses studied was limited to the region of south-western Poland. Therefore, generalizing the results to all nurses in the country and abroad should be done with caution. Secondly, the study is of a crosssectional design; thus, it does not measure many variables of organizational type, management circumstances, leadership style, nor relationships with other members of the medical team. Moreover, in further studies, it would be necessary to extend the research tools, consider a broader spectrum of sociodemographic and occupational variables, and enlarge the study to other regions of Poland. However, despite the above limitations, this study can be considered helpful due to the comprehensiveness of the information and analyzed variables affecting occupational burnout among nursing professionals.

\section{Research Directions}

There is still a large area for research concerning occupational burnout and awareness-raising and strengthening of individual and organizational resources, which may be 
the remedy. The presented study shows that there is still a need for taking measures in this area. There is a need for further research concerning occupational burnout among nurses to develop procedures to support those at risk or those with already identified symptoms of occupational burnout. Future research should also consider the essential issues of work organization, management of potential and competencies, the leadership style of managers, and team relationships with both supervisors and nursing and medical co-workers. Future research should also include investigating the relationship between occupational burnout in nurses and reduced job productivity. This directly impacts the quality of patient care and generates potential costs to the health care system.

\section{Conclusions}

Occupational burnout is related to individual, interpersonal and organizational feelings. There is an interrelationship between personality traits, where a higher level of occupational burnout was found among nurses with a pessimistic attitude. Higher life satisfaction and an optimistic attitude to life are key factors that positively affect occupational burnout among nurses. Grater burnout is observed in nurses working in inpatient health care, shift nurses, and those with less than 20 hours of free time per week. Place of work and life orientation are identified as crucial predictors of higher burnout among nurse professionals.

Health programs should be implemented to identify and eliminate burnout through mental health support, improved communication skills, optimized teamwork, and evidence-based interventions such as yoga, meditation, mindfulness, and motivational training. It would be helpful for policymakers, managers, and researchers to implement nationwide solutions and programs for burnout prevention. These programs can provide organizations with a systems-based and proactive method of prevention, as well as a holistic and effective way to manage burnout in nursing professionals.

\section{Abbreviations}

DEP, depersonalization; EE, emotional exhaustion; JD-R, job demands-resources model; JSS, Job Satisfaction Scale; LOT-R, Life Orientation Test-Revised version; MBI, Maslach Burnout Inventory; PA, personal accomplishments; SWLS, Satisfaction with Life Scale.

\section{Acknowledgments}

There were no contributors to the article other than the authors as well as there was no writing assistance regarding our paper. The certified English language services were provided.

\section{Funding}

This research did not receive any specific grant from funding agencies in the public, commercial, or not-forprofit sectors.

\section{Disclosure}

The authors report no conflicts of interest for this work.

\section{References}

1. Pines A, Aronson E. Career Burnout: Causes and Cures. Free Pr; 1989.

2. Koutsimani P, Montgomery A, Georganta K. The relationship between burnout, depression, and anxiety: a systematic review and meta-analysis. Front Psychol. 2019;10. doi:10.3389/fpsyg.2019.00284

3. Demerouti E, Bakker AB, Nachreiner F, Schaufeli WB. The job demands-resources model of burnout. J Appl Psychol. 2001;86 (3):499-512. doi:10.1037/0021-9010.86.3.499

4. De Hert S. Burnout in healthcare workers: prevalence, impact and preventative strategies. Local Reg Anesth. 2020;13:171-183. doi:10.2147/LRA.S240564

5. Demerouti E, Bakker AB, Nachreiner F, Schaufeli WB. A model of burnout and life satisfaction amongst nurses. $J$ Adv Nurs. 2000;32 (2):454-464. doi:10.1046/j.1365-2648.2000.01496.x

6. Bakker AB, Demerouti E, Euwema MC. Job resources buffer the impact of job demands on burnout. J Occup Health Psychol. 2005;10 (2):170-180. doi:10.1037/1076-8998.10.2.170

7. Darban F, Balouchi A, Narouipour A, Safarzaei E, Shahdadi H. Effect of communication skills training on the burnout of nurses: a Cross-Sectional Study. J Clin Diagn Res. 2016;10(4):IC01-IC04. doi:10.7860/JCDR/2016/19312.7667

8. Sęk H. Cognitive appraisal and competence as determinants of burnout in helping professions. Adv Psychiatr Neurol. 2005;14(2):93-98.

9. Bartosiewicz A, Łuszczki E, Dereń K. Personalized nursing: how life satisfaction and occupational burnout influence new competences of Polish nurses. J Pers Med. 2020;10:2. doi:10.3390/jpm10020048

10. Maslach C, Jackson SE. The measurement of experienced burnout. J Organ Behav. 1981;2(2):99-113. doi:10.1002/job.4030020205

11. Maslach C, Schaufeli WB, Leiter MP. Job burnout. Annu Rev Psychol. 2001;52:397-422. doi:10.1146/annurev.psych.52.1.397

12. Maslach C. Job burnout: new directions in research and intervention. Curr Dir Psychol Sci. 2003;12(5):189-192. doi:10.1111/14678721.01258

13. Maslach C, Leiter MP. Early predictors of job burnout and engagement. J Appl Psychol. 2008;93(3):498-512. doi:10.1037/ 0021-9010.93.3.498

14. Woo T, Ho R, Tang A, Tam W. Global prevalence of burnout symptoms among nurses: a systematic review and meta-analysis. J Psychiatr Res. 2020;123:9-20. doi:10.1016/j.jpsychires.2019.12.015

15. De la Fuente-solana E, Suleiman-Martos N, Pradas-Hernández L, Gomez-Urquiza J, Cañadas-de la Fuente G, Albendín-García L. Prevalence, related factors, and levels of burnout syndrome among nurses working in gynecology and obstetrics services: a systematic review and meta-analysis. Int J Environ Res Public Health. 2019;16 (14):2585. doi:10.3390/ijerph16142585 
16. De la Fuente-solana E, Pradas-Hernández L, Ramiro-Salmerón A, et al. Burnout syndrome in paediatric oncology nurses: a systematic review and meta-analysis. Healthcare. 2020;8:3. doi:10.3390/ healthcare 8030309

17. Molina-Praena J, Ramirez-Baena L, Gómez-Urquiza JL, Cañadas GR, De la Fuente EI, Cañadas-de la Fuente GA. Levels of burnout and risk factors in medical area nurses: a Meta-Analytic Study. Int $J$ Environ Res Public Health. 2018;15(12):2800. doi:10.3390/ijerph15122800

18. Salvagioni DAJ, Melanda FN, Mesas AE, González AD, Gabani FL, de Andrade SMD. Physical, psychological and occupational consequences of job burnout: a systematic review of prospective studies. PLoS One. 2017;12(10):e0185781. doi:10.1371/journal.pone.0 185781

19. Tselebis A, Moulou A, Ilias I. Burnout versus depression and sense of coherence: study of Greek nursing staff. Nurs Health Sci. 2001;3 (2):69-71. doi:10.1046/j.1442-2018.2001.00074.X

20. Zhang X, Song Y, Jiang T, Ding N, Shi T. Interventions to reduce burnout of physicians and nurses. Medicine. 2020;99:26. doi:10.1097/MD.0000000000020992

21. Dall'Ora C, Ball J, Reinius M, Griffiths P. Burnout in nursing: a theoretical review. Hum Resour Health. 2020;18. doi:10.1186/ s12960-020-00469-9

22. Pasikowski T. Polish adaptation of Maslach Burnout Inventory questionnaire. In: Occupational Burnout. Causes. Mechanisms. Prevention. Polish Scientific Publishers PWN; 2006:13-31, 135-148.

23. Scheier MF, Carver CS, Bridges MW. Distinguishing optimism from neuroticism (and trait anxiety, self-mastery, and self-esteem): a reevaluation of the life orientation test. J Pers Soc Psychol. 1994;67(6):1063-1078. doi:10.1037/0022-3514.67.6.1063

24. Poprawa R, Juczyński Z. Adaptation of the Life Orientation Test (LOT-R). In: Measurement Tools in Health Promotion and Psychology. 2 ed. Psychological Test Laboratory of the Polish Psychological Association; 2009.

25. Diener E, Emmons RA, Larsen RJ, Griffin S. The satisfaction with life scale. J Pers Assess. 1985;49(1):71-75. doi:10.1207/ s15327752jpa4901_13

26. Juczyński Z. Adaptation of the Satisfaction With Life Scale (SWLS). In: Measurement Tools in Health Promotion and Psychology. 2 ed. Psychological Test Laboratory of the Polish Psychological Association; 2009.

27. Marć M, Bartosiewicz A, Burzyńska J, Binkowska-Bury M, Januszewicz P. The quality of life and readiness of Polish nurses to take new competences of drug prescribing. Medicine. 2018;97(36): e12129. doi:10.1097/MD.0000000000012129

28. Zalewska A. Job satisfaction scale: measurement of the cognitive aspect of overall job satisfaction. Folia Psychologica. 2003;7. Available from: http://bw.swps.edu.pl/info.seam?id=IMPORTARTICLE-00006879. Accessed March 26, 2018.

29. Sharma SK, Mudgal SK, Thakur K, Gaur R. How to calculate sample size for observational and experimental nursing research studies? Natl J Physiol Pharm Pharmacol. 2020;10(1):1-8.

30. R Core Team. R: A Language and Environment for Statistical Computing. $R$ Foundation for Statistical Computing; 2017. Available from: https://www.R-project.org/. Accessed October 22, 2021.

31. Pawlik J, Schneider-Matyka D, Jurczak A, Szkup M, Grochans E. Assessment of satisfaction of nurses working in Poland and Norway. Hygeia Public Health. 2017;52(3):249-254.

32. Wilczek-Różyczka E, Zaczyk I. Occupational burnout of Polish nurses - meta-analysis of studies. Hygeia Public Health. 2015;50 (1):9-13

33. Roczniewska M, Bakker AB. Burnout and self-regulation failure: a diary study of self-undermining and job crafting among nurses. J Adv Nurs. 2021;77(8):3424-3435. doi:10.1111/jan.14872
34. Bakker AB. Job crafting among health care professionals: the role of work engagement. J Nurs Manag. 2018;26(3):321-331. doi:10.1111/ jonm. 12551

35. The Supreme Council of Nurses and Midwives. Securing Polish society in the services of nurses and midwives. The Supreme Council of Nurses and Midwives; 2017. Available from: https:// nipip.pl/raport-naczelnej-rady-pielegniarek-poloznychzabezpieczenie-spoleczenstwa-polskiego-swiadczenia-pielegniarekpoloznych-2017-r/. Accessed October 22, 2021.

36. Ben-Harush A, Shiovitz-Ezra S, Doron I, et al. Ageism among physicians, nurses, and social workers: findings from a qualitative study. Eur J Ageing. 2016;14(1):39-48. doi:10.1007/s10433-016-0389-9

37. Marć M, Bartosiewicz A, Burzyńska J, Chmiel Z, Januszewicz P. A nursing shortage - a prospect of global and local policies. Int Nurs Rev. 2019;66(1):9-16. doi:10.1111/inr.12473

38. Tarcan M, Hikmet N, Schooley B, Top M, Tarcan GY. An analysis of the relationship between burnout, sociodemographic and workplace factors and job satisfaction among emergency department health professionals. Appl Nurs Res. 2017;34:40-47. doi:10.1016/j. apnr.2017.02.011

39. Zhu T, Zhang SS, Chen DY, et al. [Job burnout and related influencing factors in community medical staff in Nanchong, China]. Zhonghua Lao Dong Wei Sheng Zhi Ye Bing Za Zhi. 2016;34 (12):928-930. Chinese. doi:10.3760/cma.j.issn.1001-9391.201 6.12 .011

40. Patel RS, Bachu R, Adikey A, Malik M, Shah M. Factors related to physician burnout and its consequences: a review. Behav Sci. 2018;8 (11). doi:10.3390/bs8110098

41. Rudman A, Arborelius L, Dahlgren A, Finnes A, Gustavsson P. Consequences of early career nurse burnout: a prospective long-term follow-up on cognitive functions, depressive symptoms, and insomnia. EClinicalMedicine. 2020;27:100565. doi:10.1016/j. eclinm.2020.100565

42. Nowak-Starz G, Kozak B, Zdziebło K. The influence of stress connected with professional work on the occurrence of burnout syndrome in nurses working in surgical and medical treatment wards. Med Stud. 2013;29(1):7-21. doi:10.5114/ms.2013.39183

43. Hoffman AJ, Scott LD. Role stress and career satisfaction among registered nurses by work shift patterns. J Nurs Adm. 2003;33 (6):337-342. doi:10.1097/00005110-200306000-00006

44. Dall'Ora C, Griffiths P, Ball J, Simon M, Aiken LH. Association of $12 \mathrm{~h}$ shifts and nurses' job satisfaction, burnout and intention to leave: findings from a cross-sectional study of 12 European countries. BMJ Open. 2015;5:9. doi:10.1136/bmjopen-2015-008331

45. Kishi R, Kitahara T, Masuchi A, Kasai S. Work-related reproductive, musculoskeletal and mental disorders among working women-history, current issues and future research directions. Ind Health. 2002;40(2):101-112. doi:10.2486/indhealth.40.101

46. Kędra E, Sanak K. Stress and burnout in nurses. Nurs Public Health. 2013;3(2):119-132.

47. Marcysiak M, Dąbrowska O, Marcysiak M. Burnout in a nursing profession versus coping. Nurs Probl. 2014;22(3):312-318.

48. Haor B, Głowacka M, Ślusarz R, Piotrowska A. Behaviors and experiences of nurses connected with professional work. Nurs Probl. 2014;22(2):142-146.

49. Al-Turki HA, Al-Turki RA, Al-Dardas HA, et al. Burnout syndrome among multinational nurses working in Saudi Arabia. Ann Afr Med. 2010;9(4):226-229. doi:10.4103/1596-3519.70960

50. Sodomska A, Lomper K, Rycombel A, Uchmanowicz I. The professional burnout in nursing. Contemp Nurs Public Health. 2014;2:29-33.

51. Cybulska A, Trembecka J, Śmigielska A, Grochans E. Interpersonal skills of anaestetical nurses and burnout. Nurs Probl. 2017;25 (3):149-153. doi:10.5603/PP.2017.0025

52. Dębska G, Cepuch G. Occupational burnout among nurses working in primary health care institutions. Probl Nurs. 2008;16(3):273-279. 


\section{Publish your work in this journal}

Psychology Research and Behavior Management is an international, peer-reviewed, open access journal focusing on the science of psychology and its application in behavior management to develop improved outcomes in the clinical, educational, sports and business arenas. Specific topics covered in the journal include: Neuroscience, memory and decision making; Behavior modification and management; Clinical applications; Business and sports performance management; Social and developmental studies; Animal studies. The manuscript management system is completely online and includes a very quick and fair peer-review system, which is all easy to use. Visit http://www. dovepress.com/testimonials.php to read real quotes from published authors. 